\title{
Estudio y detección de factores de riesgo en la infancia: un estudio retrospectivo a través del cuestionario LISMEN
}

Research study and identification of risk factors in childhood:

a retrospective study through the LISMEN questionnaire

Marina Capitán Llamas ${ }^{a}$, Jordi Artigue Gómez ${ }^{b}$

a) Graduada en Psicología - Universidad de Barcelona

a) Psicólogo Clínico. Profesor Asociado de Psicología - Universidad de Barcelona

Correspondencia: Jordi Artigue Gómez (jordiartigue@ub.edu)

Recibido: 24/10/2019; aceptado con modificaciones: 25/05/2020

Resumen: Las relaciones de apego y la crianza en la infancia condicionan el desarrollo emocional y la aparición o no de trastornos mentales. Algunas experiencias adversas en la infancia se consideran factores de riesgo de salud en general y de salud mental en particular. Se sabe que dichas adversidades tienen un reflejo en el sistema fisiológico y provocan cambios epigenéticos en el ADN. Se realiza un estudio retrospectivo en una muestra de 29 pacientes ya diagnosticados de trastorno mental grave en la edad adulta, la mayoría hombres, de edad media de 25,2 años. Su finalidad es observar cuáles son los factores de riesgo encontrados con mayor frecuencia. Estos fueron: depresión y/o estrés de la madre durante el embarazo, separaciones tempranas y prolongadas de las figuras de cuidado, y padre emocionalmente ausente. Todos ellos afectan a los procesos de vinculación y relación dentro de la familia. Se concluye que este tipo de estudios son necesarios, ya desde la infancia, para detectar y prevenir alteraciones en las relaciones de apego que ocasionarán cambios emocionales y fisiológicos que condicionarán la salud mental de la persona.

Palabras clave: factores de riesgo, psicosis, apego, infancia. 


\begin{abstract}
Attachment relationships and upbringing during childhood condition emotional development and incidences of mental disorders or lack thereof. Some adverse experiences in childhood are considered general health risk factors and mental health risk factors in particular. Said adversities are known to be reflected in the physiological system and to provoke epigenetic changes in the DNA. A retrospective study was conducted in a sample of 29 patients who had been diagnosed with severe mental disorders during adulthood. The majority of them were male, and their average age was 25.2 years old. Risk factors found at higher rates were: mother's depression and/or stress during pregnancy, early and prolonged separations of care figures, and emotionally absent fathers. These adversities affect the processes of attachment and relationships within the family. We conclude that these kinds of studies are necessary, starting in childhood, to detect and prevent alterations in attachment relationships that will cause emotional and physiological changes that will condition the person's mental health.
\end{abstract}

Key words: risk factors, psychosis, attachment, childhood.

\title{
INTRODUCCIÓN
}

$\mathrm{E}^{\mathrm{s}}$ CIERTO QUE LA GENÉTICA JUEGa UN PAPEL IMPORTANTE en la predisposición de padecer trastornos mentales, en especial trastornos mentales graves como la esquizofrenia (1-3). No obstante, se sabe que los factores ambientales juegan un papel fundamental desde los estudios de Tienari et al. (4), cuando compararon la incidencia de la esquizofrenia en jóvenes adoptados, unos cuya madre biológica padecía esquizofrenia y otros que no tenían antecedentes de trastorno mental. Los resultados mostraron que el riesgo de padecer esquizofrenia era más alto en los niños con antecedentes familiares; sin embargo, este aumento de la incidencia solo se halló en familias con un funcionamiento que se había diagnosticado como alterado. Por lo tanto, podemos afirmar que el ambiente regula la expresión genética a través de los acontecimientos traumáticos (5-7) y que además existen una serie de factores protectores y factores de riesgo (8) que también condicionan la expresión final del trastorno.

En este estudio nos centraremos en identificar dichos factores de riesgo en un grupo de pacientes determinado. Además, expondremos algunas hipótesis sobre los posibles mecanismos responsables del paso de los factores de riesgo a las manifestaciones en la salud de las personas.

En los últimos años se han acumulado muchas evidencias de que la experiencia de la adversidad en la infancia de una persona puede tener repercusiones a más largo plazo en su salud y bienestar. Los resultados del estudio de McCrory et al. (9) 
indican que los primeros años de vida pueden representar un período sensible o crítico en el desarrollo de una enfermedad o trastorno. La experiencia de adversidad durante la infancia se asoció con un mayor riesgo de enfermedad crónica en la edad adulta: enfermedades cardiovasculares, enfermedades pulmonares y trastornos de salud mental. En particular, los que experimentaron cualquier tipo de adversidad tenían casi el doble de riesgo de padecer trastornos mentales $(11,4 \%)$ en comparación con los que no estaban expuestos $(6,6 \%)$. Se encontró que los cuatro factores evaluados que fueron significativos como factores de riesgo fueron: abuso de sustancias en la familia, abuso físico infantil, abuso sexual infantil y situación económica desfavorable, que fue el que tuvo una significación menor en comparación con los tres primeros. Es importante destacar que el riesgo relativo aumentaba considerablemente cuando los factores de riesgo pasaban a ser de uno $(1,28)$ a dos o más de dos $(2,31)$, lo que nos indica que el trastorno puede darse por la acumulación de factores y no por uno en concreto. Esto está relacionado con el término sensibilización conductual (10), que muestra cómo la exposición repetida a diversos estresores psicosociales aumenta las respuestas conductuales, neuroquímicas o psicométricas a una exposición posterior a un nuevo factor estresante psicosocial, incluso si esta exposición posterior no es tan grave como la anterior. Sabemos que el riesgo de padecer un trastorno mental aumenta con el número de factores de riesgo, no obstante, la especificidad del trauma en relación a la posible aparición de un trastorno mental determinado sigue siendo confusa (11).

En el estudio de Kessler et al. (12) se estudiaron los factores de riesgo en una muestra de 51.945 adultos de nueve países clasificados por el Banco Mundial como de altos ingresos (Bélgica, Francia, Alemania, Israel, Italia, Japón, Países Bajos, Espańa y EE. UU.), seis de alto- medio (Colombia, India, Iraq, Nigeria, República Popular de China, Ucrania) y seis de ingresos medios-bajos (Brasil, Bulgaria, Líbano, México, Rumania y Sudáfrica). Las 12 adversidades infantiles siguientes se asociaron significativamente con un riesgo elevado de aparición de trastornos del DSM-IV en todos los países: muerte de un progenitor, divorcio de los padres, trastorno mental en los padres, consumo de substancias parental, comportamiento criminal de los padres, violencia familiar, abuso físico, abuso sexual, negligencia parental, enfermedad física del niño y situación económica desfavorable.

Podemos considerar que existen factores de riesgo distales de diferente tipo y gravedad, como los acontecimientos adversos en la infancia, que afectarán, de diferente forma, según el niño y su entorno, a su salud mental. Por ejemplo, el menor nivel educativo puede conducir a un futuro empleo precario y así determinar peores condiciones materiales en la edad adulta, que aumentan el riesgo de implicarse en una variedad de comportamientos que comprometen la salud. En ambientes sociales de riesgo se pueden desarrollar diferentes estilos de afrontamiento, estrategias de regulación emocional 
y cogniciones sociales que pueden llegar a ser disfuncionales y aumentar el estrés (9). Por consiguiente, sabemos que este aumento del estrés lleva consigo un incremento del riesgo de psicosis, en especial en el caso de la exposición acumulada (10).

Se han investigado en nuestro entorno las diferencias de aparición de un trastorno mental grave como la psicosis en dos comunidades diferenciadas por ser o no ambiente social de riesgo. En un estudio transversal realizado en Barcelona con una muestra de 21.536 pacientes, se comparó el número de personas diagnosticadas de esquizofrenia y otras psicosis en dos barrios de la ciudad, uno de ellos caracterizado por la presencia de diversos factores de riesgo psicosociales. Se halló que la prevalencia de esquizofrenia y otras psicosis en el barrio que presentaba más factores de riesgo era alrededor del doble que la prevalencia en el otro barrio (13).

Desde un punto de vista biológico, existen evidencias de que la experiencia de la adversidad en la infancia puede inducir cambios en los sistemas fisiológicos que influyen en el riesgo de enfermedad en la vida posterior. Estos se basan en los cambios en una serie de parámetros biológicos. La respuesta neurobiológica al estrés induce la activación en dos vías neuroendocrinas -el sistema nervioso simpático y el eje hipotalámico-hipófisis-adrenal- $(10,14)$, que son centrales para la respuesta al estrés. La activación crónica de estos sistemas puede causar desequilibrios en la serie de mediadores bioquímicos implicados en el inicio, mantenimiento e inhibición de la respuesta al estrés. Como consecuencia, estos sistemas fisiológicos se vuelven crónicamente desregulados en el tiempo, llevando a un mayor desgaste y una mayor probabilidad de sufrir un trastorno (9).

Uno de los mecanismos más estudiados actualmente en este ámbito, la epigenética, da la respuesta a cómo la información genética se regula sin cambiar la secuencia de ADN (15). De esta manera, la exposición a eventos aversivos de forma temprana en la vida, como el estrés materno y el comportamiento de los padres en la infancia del individuo, puede llevar a cambios epigenéticos como las modificaciones de las histonas y la metilación del ADN. Los fenotipos conductuales relacionados con el estrés y la psicopatología están correlacionados con la metilación alterada del ADN de varios genes, incluidos los reguladores del eje hipotalámico-hipófisis-adrenal, mencionado anteriormente, que es un foco principal de la epigenética para la investigación pediátrica en salud (16). Los cambios epigenéticos relacionados con la adversidad se observan en el tejido cerebral, pero también en el tejido periférico, lo que apoya aún más el impacto de la adversidad temprana en todo el organismo (15). Se han descrito varias variantes genéticas o puntuaciones de riesgo poligénico que interactúan con la adversidad temprana, algunas con hallazgos relativamente consistentes, como por ejemplo polimorfismos en el promotor del gen transportador de serotonina, el CRHR1 y el gen FKBP5. Todas estas variantes no solo han demostrado interaccionar con la adversidad para predecir la psicopatología, sino también con 
los fenotipos intermedios, como los cambios cerebrales estructurales o funcionales o las respuestas endocrinas (15).

Los estudios con animales sugieren que los factores estresantes en la infancia modifican significativamente varias marcas epigenéticas en múltiples genes y regiones. Estos factores pueden ser la exposición materna a fármacos, estrés y tóxicos, lo que puede contribuir a una alteración del epigenoma y puede conducir a un déficit en el desarrollo neurológico y del comportamiento del pequeño. Además, también se sabe que las variaciones en la atención y el cuidado materno se relacionan con cambios en la metilación en el gen del receptor de glucocorticoides (NR3C1) (17). Por otro lado, los estudios con humanos apuntan que algunos de los factores que influyen en los perfiles de metilación son: el abuso infantil, el maltrato infantil, la negligencia de los padres, el abandono, la psicopatología materna, los comportamientos criminales y el consumo de substancias. También influyen otros factores en la crianza no tan extremos, como el entorno social, que incluye los barrios y los grupos sociales en situaciones de riesgo, aunque no se sabe en qué medida los cambios son permanentes. Es importante mencionar que se ha visto que la mayoría de los estudios señalan que los cambios epigenéticos en la expresión de los genes aumentan la probabilidad de depresión en la vida adulta (16).

\section{MATERIAL Y MÉTODOS}

El objetivo principal es estudiar la presencia y frecuencia de factores de riesgo en pacientes ya diagnosticados de trastorno mental. Dicho objetivo se lleva a cabo mediante una evaluación retrospectiva de los factores de riesgo presentes en la infancia y la adolescencia del individuo. Para ello se realiza un análisis descriptivo de los resultados obtenidos y se elabora un análisis cualitativo de los factores de riesgo presentes en las personas evaluadas.

El tipo de diseño utilizado es transversal, ya que las variables se analizan en el momento en que el paciente es reclutado, y retrospectivo, puesto que se exploran variables que tuvieron lugar en su infancia y adolescencia.

\section{a) Muestra}

Para llevar a cabo los objetivos del proyecto se utilizan los datos existentes de pacientes provenientes de dos centros, uno del sector público y otro del sector privado, 17 y 12 casos de cada centro, respectivamente; en total, 29 personas de edad media de 25,2 años.

Se incluyeron en el estudio todos los pacientes diagnosticados de trastorno mental con historia clínica abierta en el momento del estudio, con información objetivable sobre la presencia de factores de riesgo. La recogida de datos se realizó 
mediante un muestreo acumulativo, creando un registro de los datos de cada caso que entra a formar parte de la muestra.

\section{b) Material}

Para la realización de la exploración biográfica retrospectiva sobre la presencia de acontecimientos vitales considerados como factores de riesgo para la salud mental se utilizó el cuestionario de Listado de Ítems en Salud Mental (LISMEN) $(18,19)$. El cuestionario cuenta con 89 variables que evalúan múltiples factores de riesgo, y estas configuran una base de datos junto a otras variables de interés que se exponen a continuación:

Identificador.

Sexo.

Fecha de nacimiento.

Factores de riesgo: Los factores de riesgo son las 89 variables LISMEN. Cada variable se registra según el rango de edad (0-2 años, 3-5 años, 6-11 años y 12-17 años) y de cada etapa hay tres tipos de respuesta: -1 : Ausencia, no hay presencia de este ítem; 0: No se dispone de la información suficiente; 1: Presencia del factor de riesgo.

\section{c) Procedimiento}

Se administraron los cuestionarios a los pacientes diagnosticados de trastorno mental grave (trastornos del espectro de la psicosis y en estado mental de alto riesgo de psicosis) como parte del proceso de exploración. La forma de administración fue mediante una entrevista con el paciente, acompańado en la mayoría de los casos de la familia. En ella se extraía la información necesaria en cuanto a factores de riesgo y a las variables sociodemográficas. Posteriormente se introdujo toda la información recogida en una base de datos con la que se trabaja para extraer los resultados que se exponen a continuación.

Dicha base de datos ha sido diseñada para no obtener medidas repetidas por grupos de edad. Esta consta de una columna para los códigos, otra para la fecha de nacimiento, otra para el diagnóstico y otra para el sexo. También incluye 89 columnas, una para cada ítem del cuestionario. Por otro lado, existe una columna del total de casillas con respuesta, que es 89 en todos los casos, lo que muestra que se respondieron todos los ítems de todos los cuestionarios. Por último, existen tres columnas de resultados: una indica la presencia del factor de riesgo (1); otra, la no información (0); y la última, la ausencia del factor de riesgo (-1). En cuanto a las filas, hay una fila para cada sujeto, más una última que indica el total de presencia de cada ítem. 
Como se ha comentado, el cuestionario empleado en la muestra de 29 pacientes fue el LISMEN. En su estudio de sensibilidad y validación se estableció el punto de corte en 10 (18). Por lo tanto, los casos con puntuaciones iguales o superiores a 10 son personas que a nivel estadístico se les sitúa en el grupo de riesgo; es decir, que han sufrido acontecimientos vitales que si no son tratados o cuidados emocionalmente pueden influenciar negativamente en su salud mental y desarrollar en algún momento de su vida un trastorno.

En un análisis cuantitativo de los resultados (Tabla 1), se observa que de los 29 pacientes tan solo dos presentan puntuaciones por debajo del punto de corte, lo cual les excluiría de pertenecer al grupo de riesgo.

Por otro lado, se realiza un análisis de tipo cualitativo sobre los resultados encontrados en este estudio piloto. En la Tabla 2 se muestran, de menor a mayor, los ítems según su frecuencia de presencia. Es importante mencionar que solo aparecen los ítems cuya frecuencia es superior al 25\%. Se ha considerado este criterio de forma arbitraria, pero en base a la observación clínica. Es decir, un factor de riesgo que aparezca con una frecuencia cercana o mayor del 25\% se debería considerar para estudiarlo en la biografía de los pacientes aunque estos pacientes no sean considerados de riesgo.

\section{Discusión}

Como era de esperar, dadas las características de la muestra, 27 de los 29 pacientes han vivido o viven una acumulación de adversidades en su vida que les coloca en una situación de riesgo para su salud mental (Tabla 1). Esto no sorprende teniendo en cuenta que todos están diagnosticados de trastorno mental. En este grupo de personas, a través del cuestionario LISMEN (18), se vuelve a evidenciar que existe la posibilidad de que las adversidades sufridas en la infancia favorezcan la expresión de alteraciones en su salud mental a través de los cambios epigenéticos, como sugieren otros estudios $(6,7,9,20)$. Aunque se desconoce el proceso que siguen dichas alteraciones y todavía se hace difícil medir la relación entre unos y otros.

Por lo que respecta a los dos pacientes que no están considerados en situación de riesgo, a pesar de tener puntuaciones muy cercanas (7 y 8), cabe la posibilidad de que se traten de falsos negativos. En este grupo de pacientes, al tener sus integrantes un diagnóstico en salud mental no existe la posibilidad de tener casos de falsos positivos.

Respecto al análisis cualitativo (Tabla 2), las cifras que reflejan mayor presencia se refieren a variables que afectan a los procesos de vinculación y relación dentro de la familia, al sufrimiento emocional y a las dificultades en la relación con los iguales. 
El factor de riesgo presente con más frecuencia es el de funciones paternales empobrecidas y forma parte de las variables que estudian la vinculación y las relaciones familiares: se puntúa cuando en una familia existe la figura del padre y este, a nivel emocional, no proporciona afecto ni es empático; es decir, no se puede ilusionar con la crianza y educación de su hijo o hija. Como ya se ha definido hace años desde la teoría del apego, se trata de una incapacidad del adulto de sostener en su mente una representación del niño como alguien que tiene sentimientos propios (21). La situación extrema se produce cuando abandona sus responsabilidades en la crianza. Aunque a veces se igualen las situaciones de abandono a separaciones y divorcios, no siempre es así. Se diferencia entre valoración emocional y situación vital de la pareja de adultos. Se habla de figura del padre y no de padre biológico porque en determinados hogares y situaciones familiares no se pueden asimilar estas circunstancias. Más importante que la definición del porqué aquel hombre está vinculado o no a un niño o niña es la cualidad del vínculo que establece.

Con menor frecuencia aparecen las funciones maternales empobrecidas; en cambio, con frecuencia más alta encontramos las dificultades de relación de la madre con su hijo o hija. Estos hallazgos son congruentes con los resultados de algunas revisiones bibliográficas (8), donde se relacionan con el aumento del riesgo de padecer alteraciones emocionales, aunque por sí solas no determinan dichas alteraciones.

Las dificultades en los procesos de vinculación durante la crianza pueden originarse en circunstancias adversas, como la presencia de problemas obstétricos, dificultades en la lactancia, retraimiento en la relación en los primeros meses de vida, trastornos del sueño y dificultades para dormir solo (cohabitación) cuando ya existen competencias básicas para hacerlo (Tabla 2). Estos resultados son también congruentes con las investigaciones de cohorte, cuyos hallazgos señalan que aquellos que desarrollaron un trastorno mental grave tenían en común, entre otros factores de riesgo, haber padecido problemas obstétricos (22), signos de asfixia (23), malnutrición fetal (24) y retraimiento en la relación (25). Son investigaciones ya clásicas, que permitieron pensar en la influencia del ambiente en el neurodesarrollo del bebé y el niño, es decir, de la epigenética.

A través del cuestionario LISMEN se exploran también otras circunstancias adversas que tienen consecuencias en la vinculación en los años de crianza: depresión y/o estrés de la madre durante el embarazo, separaciones tempranas y prolongadas de las figuras paternas (ya sea por hospitalizaciones, separaciones tras un conflicto o incluso la muerte inesperada de un padre). En la bibliografía científica existen estudios que analizan la relación entre este tipo de adversidades y su expresión en las emociones a través de las estructuras cerebrales (9), incluso ya desde el período intrauterino (26). El psicoanálisis propuso desde sus inicios la teoría del trauma como argumento explicativo de la génesis de los trastornos mentales y, aunque con 
muchas variantes y algunas modificaciones en su formulación, las experiencias adversas vividas, consideradas como factores de riesgo, son admitidas por los diferentes modelos teóricos como una parte de la fundamentación y comprensión del origen de los trastornos mentales. Las neurociencias están aportando en las últimas décadas evidencias sobre la existencia de un soporte biológico en el sistema cerebral donde se muestran los cambios que provocan las experiencias traumáticas o, por ejemplo, las diferencias en los niveles hormonales dependiendo de si la crianza es materna o no (14) y cómo estas se activan ante nuevos elementos traumáticos o, utilizando el símil que nos proporciona la química, precipitantes.

En cuanto al entorno próximo de los pacientes, se observa a través de la exploración que en su infancia están presentes variables relativas a una evolución emocional alterada: reacciones emocionales excesivas, mentiras reiteradas, tener menos de dos amigos, miedos frecuentes, niño raro, rabietas frecuentes. A nivel escolar, están presentes desde las dificultades hasta el fracaso pasando por el absentismo. Otras variables fueron: niño triste, dificultades en mantener y sostener la atención, y problemas frecuentes en la relación madre-hijo. Todas las variables citadas estuvieron presentes entre el $27,6 \%$ y el $48 \%$ de los casos (Tabla 2).

Se deduce que estos niños tienen dificultades en la relación con sus iguales y con su familia. Son niños y niñas expuestos a posibles problemas de aislamiento social. En los estudios de cohortes citados, estas dificultades también eran variables predictivas de riesgo de padecer una alteración en salud mental en la misma infancia (27) o en etapas posteriores (24). A la vez, unido a estas, el rendimiento en los aprendizajes también tiene ese carácter predictivo (28) cuando no se trata adecuadamente (29).

Los estilos de apego no seguro se inician desde la primera infancia y se reconocen por la presencia de miedos y ansiedades (rabietas, conductas raras, mentiras...), en especial ante las separaciones. En un estudio con 31 adolescentes y adultos jóvenes en Estado Mental de Alto Riesgo (EMAR), se comprobó la influencia que tiene el estilo de apego en el tratamiento y evolución de dichos pacientes. Se clasificó el tipo de apego con el cuestionario sobre relaciones RQ (30) y se observó, después de 12 meses de seguimiento, cómo las intervenciones terapéuticas obtenían peores resultados en las personas con un apego inseguro (ya sea del tipo temeroso, preocupado o desorganizado) $(31,32)$. De ahí la importancia que tiene para la prevención de los trastornos mentales graves en la vida adulta detectar en la infancia conductas alteradas para estudiar el tipo de apego y tratarlo, si es necesario.

Por último, la adolescencia es un período especialmente sensible a los estresores sociales (33). Pueden aparecer señales, quizás no muy llamativas, pero que se pueden entender como una conducta que nos indica la presencia de una alteración emocional del paciente: las dificultades de autonomía, la presencia de ideas raras, el 
comportamiento alimentario alterado, las ideas autorreferenciales, la fatiga crónica, la ansiedad social excesiva, el empeoramiento del funcionamiento relacional y los cambios de humor. En conjunto, es lo que técnicamente se denominan pródromos de un trastorno mental grave del espectro psicótico. Pero detectados a tiempo se puede cambiar el curso del trastorno. Cada vez son más frecuentes los estudios donde se pone de manifiesto la existencia de experiencias psicóticas subclínicas en la población en general (34) que permiten hacer prevención de dicho trastorno (35).

\section{Conclusiones}

En este estudio piloto, se ha constatado que existen diversos eventos adversos en la infancia del individuo que inciden en el posible desarrollo de los trastornos mentales. Algunos de los componentes más importantes son los estilos de relación y de crianza, las dificultades en los primeros meses de vida, las capacidades de relación y de tolerancia a la frustración en la etapa escolar y los síntomas de un funcionamiento mental alterado en la pubertad y adolescencia. Estas circunstancias alteran las relaciones de apego y contribuyen a la disregulación emocional del niño y del adolescente. Esto ocurrirá si el número de factores de riesgo acumulado es alto, si estos son graves y si la presencia de factores protectores es reducida.

Desde hace décadas existen numerosas evidencias de los beneficios y la eficacia de las intervenciones de prevención y promoción en salud mental (36). El estudio y descripción de los factores de riesgo nos permite elaborar estrategias preventivas que ayuden a las personas en su evolución o desarrollo emocional protegiéndolas del trastorno mental. Esta es la finalidad de la utilización del cuestionario LISMEN en forma de entrevista: la detección de la acumulación de factores de riesgo con finalidades preventivas. Es cierto que invertir en el tratamiento de las personas diagnosticadas es de gran importancia; no obstante, hacerlo en prevención es fundamental, ya que de esta manera se ayuda a la persona desde el primer momento e incluso se puede evitar el futuro trastorno.

\section{LiMitACIONES}

La principales limitaciones del estudio son su muestra reducida y su carácter retrospectivo. Las certezas, en el ámbito que trabajamos, no son posibles; pero existen agrupaciones de factores de riesgo que, por su frecuencia, nos hacen pensar en tendencias en cuanto al riesgo de psicopatología. Por otro lado, las agrupaciones que hemos encontrado son coincidentes con estudios publicados en este ámbito de la salud mental. 


\section{Agradecimientos}

Este trabajo se ha realizado gracias a la dotación de tiempo que facilita la Facultad de Psicología de la Universidad de Barcelona en la asignatura Trabajo Práctico que cursó Marina Capitán.

Agradecemos la colaboración de la Fundación Sanitaria St Pere Claver de Barcelona, en concreto al Centro de Salud Mental de Adultos (CSMA) de Sants.

También participaron en la recogida de la muestra profesionales que colaboran a tiempo parcial con la Asociación SAPPS (Servicio de Atención y Prevención en Salud Mental): Montse Martínez, enfermera, y Anna Oriol, trabajadora social.

\section{BiBLIOGRAFÍA}

(1) Hans SL, Auerbach JG, Styr B, Marcus J. Offspring of parents with schizophrenia: mental disorders during childhood and adolescence. Schizophr Bull. 2004;30(2):303315.

(2) Tizón JL, Parra B, Artigue J, Pareja F, Pérez C, Ferrando J, et al. Hijos de pacientes con psicosis en el proyecto SASPE: Investigar para cuidar un futuro comprometido. Arch de Psiquiatr. 2006;69(1):47-68.

(3) Goldstein JM, Buka SL, Seidman LJ, Tsuang MT. Specificity of familial transmission of schizophrenia psychosis spectrum and affective psychoses in the New England family study's high-risk design. Arch Gen Psychiatry. 2010;67(5):458-467.

(4) Tienari P, Wynne LC, Sorri A, Lahti I, Läksy K, Moring J, et al. Genotype-environment interaction in schizophrenia-spectrum disorder. Br J Psychiatry. 2004;184(3):216222.

(5) Mennigen E, Bearden C. Psychosis risk and development: what do we know from population-based studies? Biol Psychiatry. 2019. [epub ahead of print]. Disponible en: https://doi.org/10.1016/j.biopsych.2019.12.014

(6) Read J, Van Os J, Morrison AP, Ross CA. Childhood trauma, psychosis and schizophrenia: a literature review with theoretical and clinical implications. Acta Psychiatr Scand. 2005;112:330-350.

(7) Varese F, Smeets F, Drukker M, Lieverse R, Lataster T, Viechtbauer W, et al. Childhood adversities increase the risk of psychosis: a meta-analysis of patient-control, prospective- and cross-sectional cohort studies. Schizophr Bull. 2012;38(4):661671.

(8) Artigue J, Tizón JL. Una revisión sobre los factores de riesgo en la infancia para la esquizofrenia y los trastornos mentales graves del adulto. Aten Primaria. 2014;46(7):336-356.

(9) McCrory C, Dooley C, Layte R, Kenny RA. The lasting legacy of childhood adversity for disease risk in later life. Health Psychol Rev. 2015;34(7): 687-696. 
(10) Van Winkel R, Stefanis NC, Myin-Germeys I. Psychosocial stress and psychosis. A review of the neurobiological mechanisms and the evidence for gene-stress interaction. Schizophr Bull.. 2008;34(6):1095-1105.

(11) Gibson LE, Alloy LB, Ellman LM. Trauma and the psychosis spectrum: A review of symptom specificity and explanatory mechanisms. Clin. Psychol. Rev. 2016;49: 92-105.

(12) Kessler RC, McLaughlin KA, Green JG, Gruber MJ, Sampson NA, Zaslavsky AM, et al. Childhood adversities and adult psychopathology in the WHO World Mental Health Surveys. Br J Psychiatry. 2010;197(5):378-385.

(13) Tizón JL, Ferrando J, Artigue J, Parra B, Parés A, Gomá M, et al. Psicosis y diferencias sociales: Comparando la prevalencia de las psicosis en dos medios urbanos diferenciados. Rev. Asoc. Esp. Neuropsiq. 2010;30(106):193-218.

(14) Debbané M, Salaminios G, Luyten P, Badout D, Armando M, Solida Tozzi A, et al. Attachment, neurobiology, and mentalizing along the psychosis continuum. Front Hum Neurosci. 2016;10:406.

(15) Binder EB. The importance of understanding the biological mechanisms of trauma. Acta Psychiatr Scand. 2016;134(4):279-280.

(16) Mitchell C, Schneper LM, Notterman DA. DNA methylation, early life environment, and health outcomes. Pediatr Res. 2015;79(1-2):212-219.

(17) Palma-Gudiel H, Córdova-Palomera A, Leza JC, Fañanás L. Glucocorticoid receptor gene (NR3C1) methylation processes as mediators of early adversity in stress-related disorders causality: a critical review. Neurosci Biobehav Rev. 2015;55:520-535.

(18) Artigue J, Tizón J, Salamero M. Reliability and validity of the List of Mental Health Items (LISMEN). Schizophr. Res. 2016;176:423-430.

(19) Artigue J. El Lismen: un cuestionario para explorar los factores de riesgo en salud mental. Temas de Psicoanálisis 2013; 5: 1-21.

(20) Dangerfield M. Negligencia y violencia sobre el adolescente: abordaje desde un hospital de día. Temas de Psicoanálisis. 2012;4(2):1-10.

(21) Fonagy P, Steele H, Steele M. Maternal representations of attachment during pregnancy predict the organization of infant-mother attachment at one year of age. Child Dev. 1991;62:891-905.

(22) Olin SC, Mednick SA. Risk factors of psychosis: identifying vulnerable populations premorbidly. Schizophr Bull. 1996;22(2):223-240.

(23) Jones PB, Rantakallio P, Hartikainen AL, Isohanni M, Sipila P. Schizophrenia as a long-term outcome of pregnancy, delivery, and perinatal complications: a 28-year follow-up of the 1966 North Finland general population birth cohort. Am J Psychiatry. 1998;155(3):355-364.

(24) Dalman C, Allebeck P, Cullberg J, Grunewald C, Koster M. Obstetric complications and the risk of schizophrenia: a longitudinal study of a national birth cohort. Arch. Gen. Psychiatry. 1999;56(3):234-240.

(25) Guedeney A, Fermanian JA. Validity and reliability study of assessment and screening for sustained withdrawal reaction in infancy: the Alarm Distress Baby Scale. Infant Ment Health J. 2001;22(5):559-575. 
(26) Khashan AS, Abel KM, McNamee R, Pedersen MG, Webb RT, Baker PN, et al. Higher risk of offspring schizophrenia following antenatal maternal exposure to severe adverse life events. Arch. Gen. Psychiatry. 2008;65(2):146-152.

(27) Choquet M, Facy F, Laurent F, Davidson F. Discovery of risk groups in a population of children of pre-school age by method of typological analysis. Early Child Dev Care. 1982;9(3):255-274.

(28) Zabala I, Manzano J. Psicopatología durante la infancia en un grupo de adultos esquizofrénicos. Inform Psiquiátricas. 1993;134(4):381-387.

(29) Isohanni M, Lauronen E, Moilanen K, Isohanni I, Kemppainen L, Koponen H, et al. Predictors of schizophrenia: evidence from the Northern Finland 1966 birth cohort and other sources. Br J Psychiatry. 2005;187:4-7.

(30) Bartholomew K, Horowitz LM. Attachment styles among young adults. A test of a four-category model. J Pers Soc Psychol 1991;61:226-244.

(31) Quijada Y, Tizón JL, Artigue J, Kwapil TR, Barrantes-Vidal N. Attachment style predicts 6-month improvement in psychoticism in persons with at-risk mental states for psychosis. Early Interv Psychia. 2012;6(4):442-449.

(32) Quijada Y, Kwapil TR, Tizón J, Sheinbaum T, Barrantes-Vidal N. Impact of attachment style on the 1-year outcome of persons with an at-risk mental state for psychosis. Psychiatry Res. 2015;228:849-856.

(33) March-Llanes J, Marqués-Feixa L, Mezquita L, Fañanás L, Moya-Higueras J. Stressful life events during adolescence and risk for externalizing and internalizing psychopathology: a meta-analysis. Eur Child Adolesc Psychiatry 2017;26:1409-1422.

(34) Van Os J, Linscott J, Myin-Germeys I, Delespaul P, Krabbendam L. A systematic review and meta-analysis of the psychosis continuum: evidence for a psychosis proneness-persistence-impairment model of psychotic disorder. Psychol Med. 2009;39:179-195.

(35) Tizón JL, Eddy L, Ibáńez M, Parra B, Torregrosa M, De Muga L, et al. Salut mental infanto-juvenil. En: Prats B, Prats R, Plasència A, De Frutos E., Colet A (compiladores). Protocol d'activitats preventives i de promoció de la salut a l'edat pediátrica: Infancia amb salut. Barcelona: Generalitat de Catalunya, Departament de Salut, 2008; p. 127-148.

(36) Jané-Llopis E. La eficacia de la promoción de la salud mental y la prevención de los trastornos mentales. Rev. Asoc. Esp. Neuropsiq. 2004;(89):67-77. 
TABla I

Análisis cuantitativo de los resultados del LISMEN

\begin{tabular}{|c|c|}
\hline $\begin{array}{c}\text { Total } \\
\text { Presencia de Factores de Riesgo }\end{array}$ & $\begin{array}{c}\text { Núm. } \\
\text { pacientes }\end{array}$ \\
\hline $\mathbf{0 - 5}$ & 0 \\
\hline $\mathbf{6 - 9}$ & 2 \\
\hline $\mathbf{1 0}$ & 1 \\
\hline $\mathbf{1 1 - 1 5}$ & 8 \\
\hline $\mathbf{1 6 - 2 0}$ & 12 \\
\hline $\mathbf{2 1 - 2 5}$ & 3 \\
\hline $\mathbf{2 6 - 3 0}$ & 3 \\
\hline Total & 29 \\
\hline
\end{tabular}


TABla 2

Análisis cualitativo: factores de riesgo presentes en mayor frecuencia

\begin{tabular}{|c|c|c|}
\hline \multicolumn{3}{|c|}{ Alteraciones en los vínculos familiares } \\
\hline$\%$ & Factor de riesgo & $n$ \\
\hline 58,6 & Funciones paternales empobrecidas & 17 \\
\hline 55,2 & Problemas relación madre-hijo & 16 \\
\hline 51,7 & Cohabitación & 15 \\
\hline 31 & Diferencias lactancia & 9 \\
\hline 31 & Retraimiento en la relación ( $0-2$ años) & 9 \\
\hline 31 & Problemas obstétricos & 9 \\
\hline 27,6 & Depresión durante el embarazo & 8 \\
\hline 27,6 & Stress durante el embarazo & 8 \\
\hline 27,6 & Separaciones tempranas & 8 \\
\hline 27,6 & Funciones maternales empobrecidas & 8 \\
\hline 27,6 & Trastorno del sueńo (0-2 años) & 8 \\
\hline \multicolumn{3}{|c|}{ Sufrimiento emocional en la infancia } \\
\hline$\%$ & Factor de riesgo & $n$ \\
\hline 55,2 & Cambios de humor & 16 \\
\hline 48,2 & Miedos & 14 \\
\hline 44,8 & Niño triste & 13 \\
\hline 41,4 & Rabietas frecuentes & 12 \\
\hline 34,5 & Dificultades mantener atención & 10 \\
\hline 34,5 & Mentiras reiteradas & 10 \\
\hline 34,5 & Ideas autorreferenciales & 10 \\
\hline 34,5 & Fatiga crónica & 10 \\
\hline
\end{tabular}




\section{Sufrimiento emocional en la infancia}

\begin{tabular}{|c|l|c|}
\hline \multicolumn{1}{|c|}{ Factor de riesgo } & $n$ \\
\hline 34,5 & Absentismo escolar & 10 \\
\hline 31 & Fracaso escolar & 9 \\
\hline 31 & Reacciones emocionales excesivas & 9 \\
\hline 27,6 & Déficit de atención & 8 \\
\hline 27,6 & Dificultades escolares & 8 \\
\hline 27,6 & Dificultades autonomía & 8 \\
\hline 27,6 & Ideas raras & 8 \\
\hline 27,6 & Comportamiento alimentario alterado & 8 \\
\hline$\%$ & Dificultades en la relación con los iguales & $n$ \\
\hline 41,4 & Factor de riesgo & 12 \\
\hline 41,4 & Empeoramiento función relacional & 13 \\
\hline 41,4 & Menos de 2 amigos & 11 \\
\hline 37,9 & Niño raro & \\
\hline
\end{tabular}

La agrupación de factores por temas es a criterio de los autores. n: número de casos donde el ítem está presente. 